\title{
Effect of Malaria Parasite on Haematological Parameters: An Institutional Experience
}

\author{
Roopak Aggarwal ${ }^{1}$, Vineet Chaturvedi ${ }^{1}$, Sanjeev Kumar Singh ${ }^{1}$, \\ Pinki Pandey ${ }^{2}$, Savita Agarwal ${ }^{1}$, Jasvinder Singh ${ }^{3}$ \\ ${ }^{1}$ Associate Professor, Department of Pathology, ${ }^{2}$ Professor and Head, Department of Pathology, \\ ${ }^{3}$ Post Graduate $2^{\text {rd }}$ Year, Department of Pathology, Uttar Pradesh University of Medical Sciences, Saifai, Etawah
}

\begin{abstract}
Objectives: Hematological changes are among the most common complications encountered in malaria. This study analyzes and statistically evaluates the hematological changes as a diagnostic test for malaria in patient with acute febrile illness.

Method: Hundred samples were diagnosed positive by the Leishman's stained thick blood film. The data were collected from the central lab, Uttar Pradesh University of Medical Sciences, Saifai, Etawahduring May 2017 to August, 2019. Laboratory records of people suspected with malaria infection such as fever other signs and symptoms as medical doctor recommended were reviewed.

Results: The finding showed that 100 patients were diagnosed to have malaria by positive smear report by microscopy. Of these were 4 were caused by P.falciparum, whereas 96 were caused by P.vivax. No patient with co-infection of P.falciparum and P.vivax were found. The following parameters were significantly lower in malaria- infected patients; red blood cells (RBCs) count, hemoglobin ( $\mathrm{Hb}$ ), platelets count, white blood cells (WBCs) counts, neutropil, monocyte, lymphocyte and eosinophil counts, while Mean corpuscular volume, Mean corpuscular hemoglobin, Mean corpuscular hemoglobin concentration, neutrophil-lymphocyte ratio (NLR) and monocyte-lymphocyte ratio (MLR) were higher in comparison to non- malaria infected patients. Thrombocytopenia was present in $91 \%$ of malaria infected patients.
\end{abstract}

Conclusion: Patients infected with malaria exhibited important changes in most of hematological parameters with low hemoglobin, platelets, WBCs and lymphocyte counts being the most important predictors of malaria infection.

Keywords: Malaria, hematological parameters, anemia, thrombocytopenia.

\section{Introduction}

Since malaria parasites are able to attach to receptors on the red blood cell surfaces, it is expected that malaria parasite have effect on haematological parameters.

\footnotetext{
Corresponding Author:

Dr Pinki Pandey

Professor and Head, Department of Pathology, Uttar Pradesh University of Medical Sciences, Saifai, Etawah e-mail:pnkdxt@gmail.com

Ph. No.: 9458547252
}

Changes in blood cell parameters are already a well known feature of malarial infections. Malaria is a major cause of death in the tropical area of the world. Two hundred and nineteen million cases were reported worldwide in $2010^{[1]}$ Haematological changes are some of the most common complications in malaria and they play a major role in malaria pathogenesis. These changes involve the major cell types such RBC'S, leucocytes and thrombocytes ${ }^{[2-5]}$. Malaria infected patients tend to have significantly lower platelets,TLCs, RBCs and $\mathrm{Hb}$ level ${ }^{[2-4,6-8]}$.

Fever and other signs and symptoms are known to be sensitive measures of malaria infection but they lack 
specificity an positive values especially in areas where malaria is less prevalent ${ }^{[3,9]}$ and it may be difficult to distinguish the signs and symptoms of diseases from other viral or bacterial infections ${ }^{[10]}$. Typically, microscopic slide examination of peripheral blood remains the most widely used test and is the gold standard for detecting malaria infection ${ }^{[11]}$.

Aim: The objective of this study was to demonstrate the impact of plasmodium falciparium and plasmodium vivax infections as well as different parasite on blood cell parameters. The haematological parameters (Red blood cells, Leukocytes, Platelets, Haemoglobin level ( $\mathrm{Hb})$, Mean corpuscular volume (MCV), Mean corpuscular Haemoglobin (MCH), differential leucocyte count, lymphocyte- neutrophil ratio and monocyte- lymphocyte ratio of patients infected with malaria were investigated.

\section{Materials and Method}

Hundred samples were diagnosed positive by the Leishman's stained thick blood film. The data were collected from the central lab, UPUMS, Saifai during May to August, 2017-2019. Laboratory records of people suspected with malaria infection such as fever other signs and symptoms as medical doctor recommended were reviewed.

About $2 \mathrm{ml}$ of whole blood was collected from patients via the anticubital vein with sterile syringes and needles, after disinfecting the puncture site with methylated spirit. Few drops of the blood from the syringe were used to make smears (both thick and thin on two different slides respectively) for the diagnosis of malaria parasites and differential white blood cell count. The Leishman staining method was used to stain the thick blood film and the thin film after it was fixed in absolute methanol for two minutes.

The slides were examined microscopically to confirm malaria parasitemia. The rest of the blood sample was emptied into ethylene diamine tetra acetic acid (EDTA) containing 4mg of the K2 EDTA salt. The sample container was inverted several times to ensure proper mixing of the anticoagulant and the blood, for determination of the complete blood count. Blood counts were performed using Sysmex XT-1800i.

The Analyzer provided data on WBCs, $\mathrm{RBCs}, \mathrm{Hb}$ level, platelet count, MCV, MCH, RDW and five other differentials.

\section{Result}

The finding showed that 100 patients were diagnosed to have malaria by positive smear report by microscopy. Of these were 4 were caused byP.falciparum, whereas 96 were caused by P.vivax. No patient with co-infection of P.falciparum and P.vivax were found, maximum number of cases were seen in the 20-40 years age group.A leishman stained peripheral smear shows many Red cells infected with trophozoites [Figure 1(i)], Schizonts and ring forms of P.Vivax [figure 1(ii) \& 1(iii)]. Higher degree of parasitemia and RBC infected with P. Falciparum were seen [figure 2(i) \& 2(ii)].

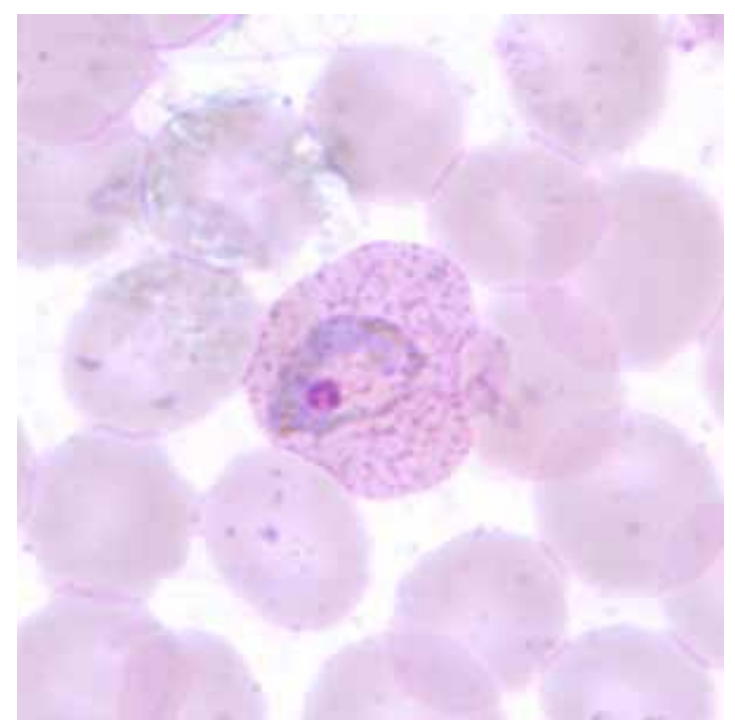

Figure 1(i): Trophozoites of Plasmodium vivax in peripheral smear (1000X) 

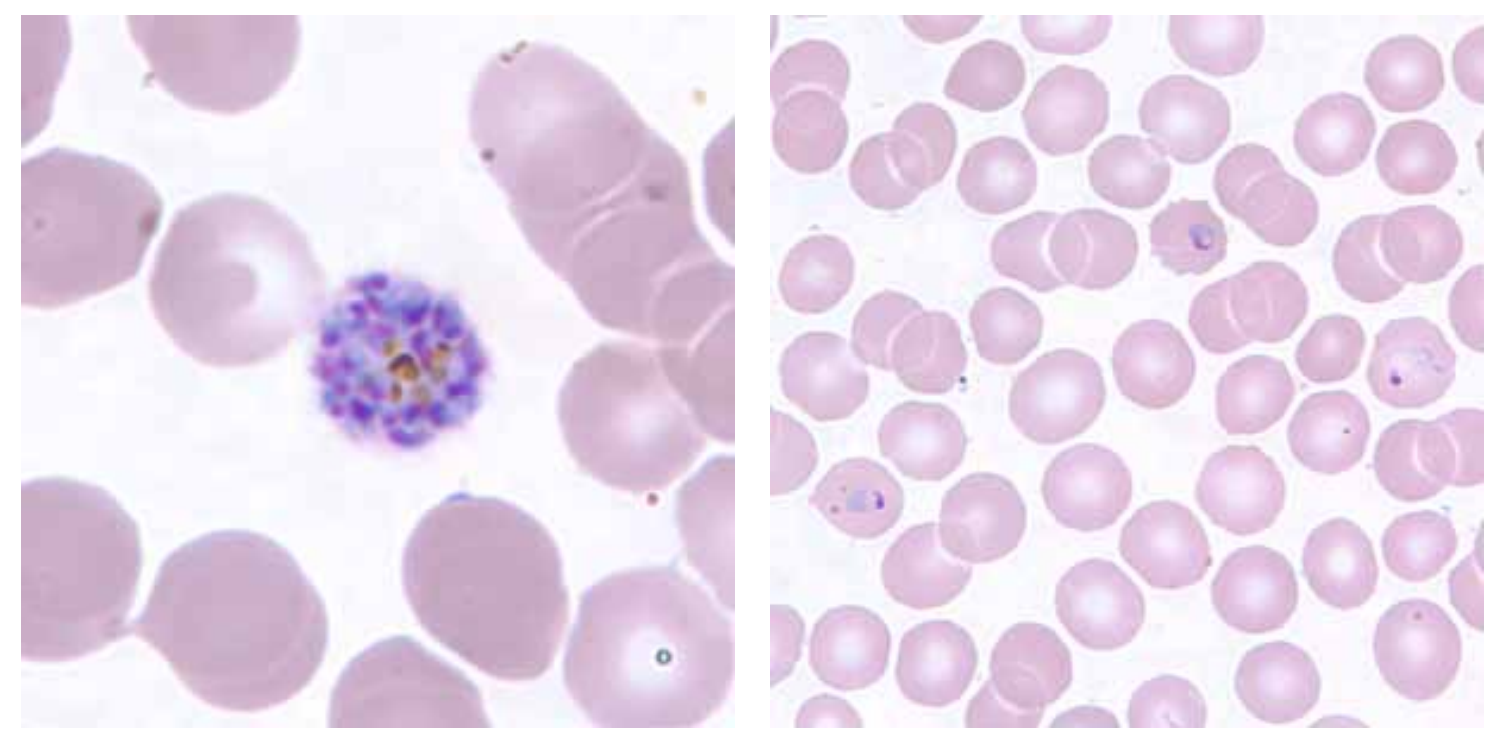

Figure 1(ii) \& Figure 1(iii): Schizonts \& Rings of P. Vivax in peripheral smear(1000X)
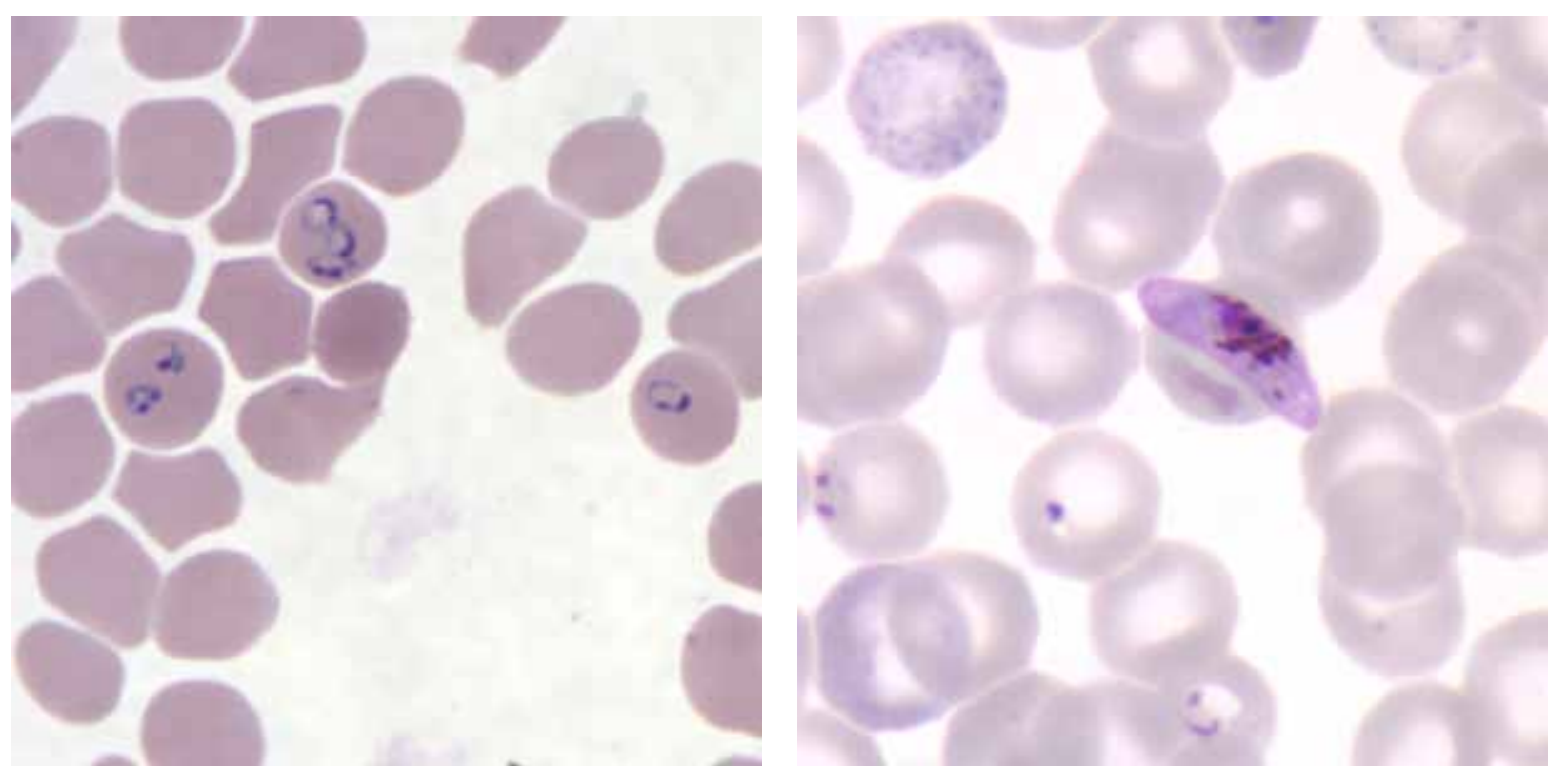

Figure 2(i) and 2(ii): Rings forms and Gametocytes of $P$.falciparum in peripheral smear(1000X)

Mean values of RBC's, WBC, Platelets and all absolute leucocyte components were significantly lower in patients with falciparum malaria compared to those with Vivax malaria and non- Malaria infected groups. Conversely, mean values of MCV, MCH, NL ratio and ML ratio were significantly higher in falciparum malaria than those with vivax malaria and non- malaria infected groups. A mean value of $\mathrm{Hb}$ was lower in patients with falciparum malaria than those with vivax and nonmalaria infected groups

Leukocyte, RBC and Platelet Counts of Patients with P.falciparum and P.vivax: Leukocyte counts were not significantly different in patients with P.falciparum malaria compared to those with P.vivax infection, for differential leukocyte counts neutrophil count was significantly higher in patients with P.falciparum compared to those with P.vivax infection. For RBC parameters, RBC count was significantly lower in patients with P.falciparum compared to those with P.vivax infection. In addition, other RBC parameters including $\mathrm{MCV}$ and $\mathrm{MCH}$ were significantly higher in patients with P.falciparum compared to those with P.vivax infection, platelet count was significantly lower in patients with P.falciparum compared to those with P.vivax. 
Table 1: Hematological values in study population

\begin{tabular}{|l|c|c|}
\hline Parameters & With Malaria Mean & Non-Malaria Mean (reference range) \\
\hline Haemoglobin (g/dl) & 11.1 & $12.8(13-18)$ \\
\hline Total leukocyte count (TLC) (10^3/cumm) & 4.1 & $8.2(4-11)$ \\
\hline Differential Leukocyte Count (DLC)(\%) & 68 & $62(40-75)$ \\
Neutrophil & 22.4 & $33(20-40)$ \\
Lymphocyte & 6.7 & $5.2(2-8)$ \\
Monocyte & 3.03 & $2.1(1.2-3.8)$ \\
\hline Neutrophil-lymphocyte ratio & 0.3 & $0.24(0.17-0.34)$ \\
\hline Monocyte-lymphocyte ratio & 3.76 & $4.42(4.2-6.5)$ \\
\hline RBC Count (million/cumm) & 34.2 & $43.8(40-54)$ \\
\hline Hematocrit (PCV)(\%) & & $86.2(82-98)$ \\
\hline Red Cell Indices & 94.7 & $29.6(27-33)$ \\
MCV (fl) & 33.9 & $172(150-400)$ \\
\hline MCH (Pg) & 78.6 & $13.8(12-15)$ \\
\hline Platelet count (10^3/cumm) & 16.4 & \\
\hline RDW\% & & \\
\hline
\end{tabular}

\section{Discussion}

This study confirms that haematological abnormalities in malaria infection are common. The findings in this study showed that during malarial infection there were peripheral blood changes such as anaemia, leucopenia and thrombocytopenia. Leucopenia was frequently seen in the malaria infected patients which was confirmed by other studies that have demonstrated leucopenia ${ }^{[6,11]}$ and contrast with other study that had demonstrate leucocytosis ${ }^{[3]}$.

Anaemia is one of the most common complications in malaria infection especially in younger children and pregnant women ${ }^{[\mathbf{1 2}]}$. The pathogenesis of anemia during malaria infection is not clearly understood. However it thought to result from the parasite's primary target on the red blood cell resulting in RBCs destruction, accelerated removal of both parasitized and non-parasitized ${ }^{[13]}$, bone marrow dysfunction ${ }^{[14]}$ and the level of parasitemia. This study reported a significant reduction of haemoglobin and $\mathrm{RBC}$ count whereas MCV, MCH level in patients infected with malaria were higher.

In addition to anemia, a reduction of platelet is another one of the well-known haematological changes observed in patients with malaria. In this study platelet count were significantly reduced in malaria infected patients. Thrombocytopenia occurred in $91 \%$ of malaria infected patients. These observations may imply that thrombocytopenia may be a marker of plasmodium infection, patients with thrombocytopenia were also likely to have anemia.

Thrombocytopenia seem to occur through peripheral destruction ${ }^{[15]}$, excessive removal of platelet by spleen pooling ${ }^{[16,17]}$ as well as platelet consumption by the process of disseminated intravascular coagulopathy (DIC) ${ }^{[18]}$. Immune mediated destruction of circulating platelets has been postulated as a cause of thrombopenia seen in malaria infection. Platelets have also been shown to mediate clumping of P.falciparum infected erythrocyte ${ }^{[19]}$. This could lead to pseudo thrombopenia, malaria infected patients have elevated levels of specific immunoglobulin $\mathrm{G}(\mathrm{IgG})$ in the blood which binds to platelet-bound malaria antigens possibly leading to accelerated destruction of platelets ${ }^{[20]}$. Platelet aggregation which is the platelet clumps are falsely counted as single platelets by the analyzer thus causing pseudo-thrombocytopenia ${ }^{[3]}$. Additionally during malaria infection, endothelial activation was activated 
and may contribute to loss of barrier function of the endothelium and organ dysfunction. This process may use platelets and their released proteins as an important regulator of endothelial permeability resulting in thrombocytopenia ${ }^{[19]}$.

\section{Conclusion}

This study can concludes that patients infected with different malarial parasites exhibit important changes and differences in many haematological parameters. The most commonly changed parametes were platelet count, $\mathrm{Hb}, \mathrm{RBC}, \mathrm{MCV}, \mathrm{MCH}, \mathrm{WBC}$, neutrophil and lymphocyte counts. Haematological investigation is relatively inexpensive and less technically sophisticated way for malaria parasite detection.

The present study has demonstrated that the haematological parameters are reliable and competent measures to diagnose severity of malaria infection, even at the early stages.

Ethical Clearance: Taken from ethical committee.

Source of Funding: Self

Conflict of Interest: Nil

\section{References}

1. WHO. World malaria report 2012. Geneva: World Health Organization; 2012.

2. Bakhubaira S. Hematological parameters in severe complicated Plasmodium falciparum malaria among adults in Aden. Turk J Haematol. 2013;30:394-399.

3. Maina RN, Walsh D, Gaddy C, Hongo G, Waitumbi J, Otieno L, Jones D, Ogutu BR. Impact of Plasmodium falciparum infection on haematological parameters in children living in Western Kenya. Malar J. 2010;9(Suppl 3):S4.

4. van Wolfswinkel ME, Vliegenthart-Jongbloed K, De Mendonca Melo M, Wever PC, McCall MB, Koelewijn R, Van Hellemond JJ, Van Genderen PJ. Predictive value of lymphocytopenia and the neutrophil-lymphocyte count ratio for severe imported malaria. Malar J. 2013; 12:101.

5. Warimwe GM, Murungi LM, Kamuyu G, Nyangweso GM, Wambua J, Naranbhai V, Fletcher HA, Hill AV, Bejon P, Osier FH, Marsh K. The ratio of monocytes to lymphocytes in peripheral blood correlates with increased susceptibility to clinical malaria in Kenyan children. PLoS One. 2013; 8:e57320.

6. Erhart LM, Yingyuen K, Chuanak N, Buathong N, Laoboonchai A, Miller RS, Meshnick SR, Gasser RA Jr, Wongsrichanalai C. Hematologic and clinical indices of malaria in a semi-immune population of western Thailand. Am J Trop Med Hyg. 2004; 70:8-14.

7. Gerardin P, Rogier C, Ka AS, Jouvencel P, Brousse $\mathrm{V}$, Imbert P. Prognostic value of thrombocytopenia in African children with falciparum malaria. Am J Trop Med Hyg. 2002; 66:686-691.

8. Adedapo AD, Falade CO, Kotila RT, Ademowo GO. Age as a risk factor for thrombocytopenia and anaemia in children treated for acute uncomplicated falciparum malaria. J Vector Borne Dis.2007; 44:266-271.

9. WHO. World Malaria Report 2013. Geneva: World Health Organization; 2013.

10. WHO. New perspective, malaria diagnosis. Geneva: World Health Organization; 2000.

11. Lathia TB, Joshi R. Can hematological parameters discriminate malaria from nonmalarious acute febrile illness in the tropics? Indian J Med Sci. 2004; 58:239-244.

12. Menendez C, Fleming AF, Alonso PL. Malariarelated anaemia. Parasitol Today. 2000;16:469476.

13. Price RN, Simpson JA, Nosten F, Luxemburger C, Hkirjaroen L, terKuile F, Chongsuphajaisiddhi T, White NJ. Factors contributing to anemia after uncomplicated falciparum malaria. Am J Trop Med Hyg. 2001;65:614-622

14. Abdalla SH. Hematopoiesis in human malaria. Blood Cells. 1990; 16:401-416.

15. Ladhani S, Lowe B, Cole AO, Kowuondo K, Newton CR. Changes in white blood cells and platelets in children with falciparum malaria: relationship to disease outcome. Br J Haematol. 2002;119:839-847.

16. Beale PJ, Cormack JD, Oldrey TB. Thrombocytopenia in malaria with immunoglobulin (IgM) changes. BMJ. 1972;1:345-349.

17. Skudowitz RB, Katz J, Lurie A, Levin J, Metz J. 
Mechanisms of thrombocytopenia in malignant tertian malaria. BMJ. 1973; 2:515-518.

18. Essien EM. The circulating platelet in acute malaria infection. Br J Haematol. 1989;72:589-590.

19. Pain A, Ferguson DJ, Kai O, Urban BC, Lowe B, Marsh K, Roberts DJ. Platelet-mediated clumping of Plasmodium falciparum-infected erythrocytes is a common adhesive phenotype and is associated with severe malaria. Proc Natl Acad Sci U S A. 2001; 98:1805-1810.

20. Moulin F, Lesage F, Legros AH, Maroga C, Moussavou A, Guyon P, Marc E, Gendrel D. Thrombocytopenia and Plasmodium falciparum malaria in children with different exposures. Arch Dis Child. 2003; 88:540-541. 